\title{
Alteración de la percepción materna sobre el estado nutricional de sus hijos ¿nuevo factor de riesgo cardiovascular?
}

\author{
Karina Rozas ${ }^{1 a}$, Patricio Huerta ${ }^{1 b}$, Jessica Planett ${ }^{1 a}$, Marylin Arancibia ${ }^{1 a}$, María Virginia Araya ${ }^{2 c}$. \\ 1.-Universidad de Tarapacá. Iquique \\ 2.-Instituto Cardiológico Iquique. \\ a.-Nutricionista. \\ b.-Mg. Salud Pública. \\ c.-Médico cardiólogo.
}

Antecedentes: La obesidad infantil es un trastorno de prevalencia creciente que predispone a la obesidad, diabetes y enfermedades cardiovasculares en el adulto. Investigaciones recientes relacionan la percepción materna con el estado nutricional real de los niños, encontrando una distorsión entre imagen corporal de sus hijos con malnutrición por exceso (MNPE).

Métodos: Estudio descriptivo de corte transversal. La población objetivo fue 320 madres de escolares de 6 a 10 años y se utilizó un muestreo por conveniencia. Se incluyeron madres de hijos con MNPE. Se evaluó la percepción de las madres a través de un pictograma. Se efectuaron mediciones antropométricas de peso, talla y perímetro de cintura (PC), presión arterial (PA), glicemia y colesterol total. En el análisis estadístico se usó Stata 14 y Epidat 4.2.

Resultados: La muestra fue conformada por 100 díadas madre/hijo con MNPE. 69\% de las madres presentaron escolaridad de enseñanza media, y $68 \%$ te- nían nivel socioeconómico bajo. Respecto de los niños, $52 \%$ eran de sexo femenino, $38 \%$ presentaban riesgo de obesidad abdominal y el $47 \%$ tenían obesidad abdominal. La glicemia en ayunas estaba alterada en 35\%, el colesterol total alterado en $12 \%$ y $31 \%$ presentaban PA elevada. El 60\% de las madres de niños con MNPE subestimaron el estado nutricional de sus hijos.

Conclusiones: En relación a los factores de riesgo cardiovascular de los escolares, un alto porcentaje presenta índices de riesgo cardiovascular elevado: obesidad abdominal, PA alterada, colesterol total y glicemias alteradas. La alteración de la percepción materna sobre el estado nutricional de sus hijos, puede repercutir en forma significativa para el desarrollo y mantenimiento de la MNPE y, por consiguiente, constituye un factor de riesgo cardiovascular, estableciendo un punto clave de intervención.

Palabras clave: Malnutrición por exceso; factores de riesgo cardiovascular; percepción materna. 


\section{Perception of child nutritional status by their mothers: a new cardiovascular risk factor?}

Background: Overrnutrition in children is increasingly more prevalent and leads to obesity, diabetes and cardiovascular disease in adults. Recent research links distorted maternal perception of nutritional status in their children with the presence of obesity in adulthood).

Methods: A descriptive cross-sectional study was performed on mothers and their schoolchildren aged 6 to 10 years old. A basic inclusion criteria was the presence of overnutrition in children and the study related maternal perception with the actual degree of overnutrition in her child. Perception was evaluated using a pictogram. Weight, height, waist circumference, blood pressure (BP), blood sugar level and total cholesterol were measured in children Stata 14 and Epidat 2.0 were used for statistical analysis.

Results: 100 mother-child pairs were studied. All children had overnutrition. $69 \%$ of mothers had high-school level education and $68 \%$ belonged in the low socio-economic stratum. There were $52 \%$ of girls. According to waist circumference $38 \%$ were at risk of malnutrition and $47 \%$ already had abdominal obesity. $35 \%$ had an altered fasting blood sugar level, $12 \%$ an elevated total cholesterol, $31 \%$ an increased blood pressure. $60 \%$ of mothers of children with overnutrition underestimated the actual nutritional status (overnutrion) of their children.

Conclusions: The presence of overnutrition and obesity indicators were high in this population. This occurred along with increased levels of BP, total cholesterol and altered blood sugar levels. The maternal perception of their children severely under estimated the level of overnutrition. This fact can have a significant impact on the development and maintenance of the excess type malnutrition and subsequent development of other cardiovascular risk factors. Therefore, improving the perception of mothers regarding the nutritional status of their children should be an important element for diminishing cardiovascular risk longterm.

Key words: malnutrition due to excess, cardiovascular risk factors, maternal perception 


\section{Introducción:}

La obesidad y el sobrepeso son los problemas de Salud Pública más graves del Siglo XXI. La obesidad en el niño es un trastorno frecuente, que predispone a la obesidad en el adulto. Es un problema que afecta tanto a países desarrollados como en vías de desarrollo, cuya etiología incluye factores genéticos y de estilos de vida no saludables, como problemas de alimentación y escasa actividad física ${ }^{1}$. La malnutrición por exceso (MNPE) en la infancia puede predisponer a desarrollar enfermedades no transmisibles como la diabetes mellitus y las enfermedades cardiovasculares ${ }^{2}$. Según la Encuesta Nacional de Salud (ENS) 2016-2017, el total de MNPE en niños menores de 8 años fue $80 \%$, mientras que en niños de 8 a 12 años fue $74 \%$ y en niños mayores de 12 años $71 \%^{3}$. El desarrollo del tejido adiposo humano se inicia durante la etapa prenatal. Existen tres períodos críticos y vulnerables para el desarrollo de la obesidad y de sus complicaciones. El primero ocurre durante la gestación y primer año de vida, el segundo entre los 5 y 7 años de edad, "período de rebote de la obesidad" y el tercero en la adolescencia. La obesidad que se desarrolla con mayor propensión en estos períodos, en especial en el "período de rebote", se caracteriza por su persistencia y complicaciones a largo plazo 4 .

En diversos estudios, se ha observado que un excesivo consumo de alimentos de alta densidad energética ricos en grasas saturadas, carbohidratos simples y sedentarismo, representan en la actualidad el estilo de vida de gran parte de los niños chilenos ${ }^{5-6}$.

El desequilibrio permanente y prolongado entre la ingestión calórica y gasto energético ha sido reconocido como la principal causa de la creciente prevalencia de obesi$\mathrm{dad}^{5-7}$. Se han desarrollado múltiples estrategias para disminuir esta patología, pero no se han logrado resultados que mejoren esta situación en la población infantil. Existen estudios que han explorado la alteración de la percepción del estado nutricional por parte de sus padres como factor de riesgo de MNPE, que demuestran que la percepción inadecuada de la proporción peso/talla en niño puede considerarse un factor de riesgo significativo para el desarrollo y/o mantenimiento del sobrepeso y obesidad, ya que $98,8 \%$ de los padres de niños con MNPE subestimaron el estado nutricional de sus hijos ${ }^{8}$. Un estudio realizado en Chile, cuyo propósito fue evaluar en población escolar la percepción de la imagen corporal (PIC) de la madre hacia sus hijos con sobrepeso u obesidad, demostró que el 54,8\% de las madres en niños obesos y el 78,3\% de las madres de niños con sobrepe- so presenta distorsión de la imagen corporal, indicando que ésta podría repercutir en la escasa adherencia al tratamiento 9 .

Nuestro trabajo plantea que la alteración de la percepción materna puede constituir un nuevo factor de riesgo cardiovascular, ya que dentro del grupo familiar son las madres las que ejercen un mayor control sobre la alimentación de sus hijos. Actúan así como un mediador importante en el tratamiento de esta patología, siendo clave su intervención para evitar a futuro el aumento de la prevalencia de la malnutrición por exceso.

\section{Metodología:}

Se realizó un estudio observacional, descriptivo, de corte transversal desde marzo a agosto del año 2018 en madres de escolares entre 6 a 10 años de primero a cuarto año básico pertenecientes al Liceo Los Cóndores de la comuna de Alto Hospicio, Región de Tarapacá.

El muestreo fue de tipo por conveniencia, que permitió seleccionar del total de la población objetivo a 320 madres/hijos, incluyendo solamente aquellas cuyos hijos presentan una MNPE. La muestra quedó conformada con 100 díadas madres/hijos.

A cada madre se le aplicó un cuestionario en donde se le solicitó indicar edad, escolaridad, ocupación y nivel socioeconómico. Según el Ministerio de Desarrollo Social de Chile se define como estrato socio económico bajo a una familia que percibe ingresos per cápita entre $\$ 0$ a \$106.214 (U\$ 0 a U\$ 143) y estrato social medio entre \$106.214 a \$301.741 (U\$ 143 a U\$ 407).

Para evaluar la percepción del estado nutricional de los escolares se formó un equipo donde participaron sicólogos clínicos, nutricionistas y estadístico, y se elaboró un pictograma de 6 figuras de imágenes corporales separado por género, equivalentes a la calificación nutricional, según la Guía Minsal 2018 de patrones de crecimiento y así evaluar el estado nutricional de niños, niñas y adolescentes, desde el nacimiento hasta los 19 años de edad. Posteriormente, el pictograma fue validado por profesionales nutricionistas pertenecientes a la Atención Primaria en Salud de Iquique. Cada imagen del pictograma corresponde a un IMC determinado y cada madre debía elegir con cual identificaba mejor a su hijo.

Los escolares se sometieron a mediciones antropométricas de peso, talla y perímetro de cintura a través de balanza digital $(\operatorname{Seca} \AA)$, tallímetro portátil $(\operatorname{Seca} \AA)$ y cinta métrica retráctil.

El diagnóstico nutricional se basó en IMC, clasificando como: riesgo de MNPE (IMC/E $\leq-1 \mathrm{DE}$ y $>-2 \mathrm{DE}$ ), 
eutrófico (IMC/E $>-1 \mathrm{DE}$ y $<+1 \mathrm{DE}$ ), sobrepeso (IMC/E $\geq+1 \mathrm{DE} y<+2 \mathrm{DE}$ ), obesidad (IMC/E $\geq+2 \mathrm{DE}$ y $<$ $+3 \mathrm{DE}$ ) y obesidad severa (IMC/E $\geq+3 \mathrm{DE})$. La medición perímetro de cintura se clasificó según sexo y edad (PC/E) como normal $(<\mathrm{p} 75)$, riesgo de obesidad abdominal ( $\geq$ p75 y $<$ p90) y obesidad abdominal ( $\geq$ p 90). Además, se midió presión arterial con monitor automático (Omron $\left.{ }^{\circledR}\right)$, se definió como glicemia un valor igual o menor a $100 \mathrm{mg} / \mathrm{dL}$ y para colesterol total aceptable en ayunas a $170 \mathrm{mg} / \mathrm{dL}$ (Accutrend $\AA$ ).

Evaluación estadística: para la descripción de las variables se utilizaron estadísticas descriptivas como promedio y desviación estándar, distribución porcentual, intervalos de confianza. Se efectuó además un análisis uni y bivariado (chi cuadrado con significación $\mathrm{p}<0.05$ ). Los análisis estadísticos se realizaron con los programas Stata 14 y Epidat 4.2.

\section{Resultados:}

Se estudiaron 100 díadas madre/hijo con MNPE. La edad promedio de las madres fue 34,08 $\pm 6,74$ años, $69 \%$ presentaba escolaridad enseñanza media, $68 \%$ pertenece a un nivel socioeconómico bajo y $55 \%$ desempeña labores de dueñas de casa.

Con respecto a los escolares, $52 \%$ era de sexo femenino, el promedio de edad fue $8,17 \pm 1,24$ años. Los promedios de los otros parámetros medidos fueron: peso corporal $35,02 \pm 8,56 \mathrm{~kg}$., talla $128 \pm 8 \mathrm{~cm}$., IMC 20,74 $\pm 2,80$ $\mathrm{kg} / \mathrm{mt} 2$, presión arterial diastólica $66,34 \pm 20,55 \mathrm{mmHg}$.,

\begin{tabular}{|l|c|c|c|}
\hline \multicolumn{5}{|c|}{ Tabla 1. Distribución de promedios población escolar. } \\
\hline & Media & DS & IC(95\%) \\
\hline Edad & 8,17 & 1,24 & $7,92-8,42$ \\
\hline Peso & 35,02 & 8,56 & $33,32-36,72$ \\
\hline Talla & 1,28 & 0,08 & $1,27-1,31$ \\
\hline IMC & 20,75 & 2,80 & $20,19-21,31$ \\
\hline Obesidad Mujeres & 22,19 & 2,09 & $21,21-23,17$ \\
\hline Obesidad Severa Mujeres & 23,72 & 3,28 & $22,48-32,95$ \\
\hline Sobrepeso Hombres & 18,49 & 0,79 & $18,15-18,82$ \\
\hline Obesidad Hombres & 21,75 & 2,05 & $20,79-22,71$ \\
\hline Obesidad Severa Hombres & 24,58 & 3,13 & $19,58-29,57$ \\
\hline PAD & 66,34 & 20,55 & $62,26-70,41$ \\
\hline PAS & 108,90 & 25,26 & $103,88-113,91$ \\
\hline Glicemia ayunas & 100,12 & 10,14 & $98,11-102,13$ \\
\hline Colesterol total & 155,45 & 14,20 & $152,62-158,27$ \\
\hline Perímetro cintura mujeres & 70,00 & 7,94 & $67,79-72,21$ \\
\hline Perímetro cintura varones & 69,96 & 8,75 & $67,42-72,51$ \\
\hline & & & \\
\hline
\end{tabular}

presión arterial sistólica 108,9 $\pm 25,26 \mathrm{mmHg}$., glicemia en ayunas $100,12 \pm 10,14 \mathrm{mg} / \mathrm{dL}$, colesterol total 155,45 $\pm 14,20 \mathrm{mg} / \mathrm{dL}$., perímetro de cintura en mujeres $70 \pm$ $7,94 \mathrm{~cm}$ y perímetro de cintura en varones $69,96 \pm 8,75$ $\mathrm{cm}$ (Tabla 1).

Se encontró $52 \%$ de sobrepeso, $40 \%$ de obesos y $8 \%$ con obesidad severa. El perímetro de cintura estaba en nivel de riesgo de obesidad abdominal y $38 \%$ estaba en riesgo de obesidad abdominal. $47 \%$ presentaba obesidad abdominal. La glicemia en ayunas estaba sobre los valores normales según norma técnica Minsal 2010; El colesterol total estaba alterado en $12 \%$ (Evaluación Nutricional del riesgo cardiovascular y metabólico INTA 2017), y 31\% tenía PA elevada (Norma Técnica Programa Nacional de la Infancia 2017). (Tabla 2 y 3).

\begin{tabular}{|c|c|c|}
\hline \multicolumn{3}{|c|}{$\begin{array}{l}\text { Tabla 2. Distribución variables sociodemográficas } \\
\text { de las madres en estudio. }\end{array}$} \\
\hline & $\mathbf{N}$ & $\%$ \\
\hline \multicolumn{3}{|l|}{ Edad } \\
\hline 20 a 39 años & 81 & 81 \\
\hline 40 a 64 años & 19 & 19 \\
\hline \multicolumn{3}{|l|}{ Estado civil } \\
\hline Casada & 40 & 40 \\
\hline Separada & 5 & 5 \\
\hline Soltera & 55 & 5 \\
\hline \multicolumn{3}{|l|}{ Escolaridad } \\
\hline Educación básica & 8 & 8 \\
\hline Educación media & 69 & 69 \\
\hline Educación técnico o profesional & 23 & 23 \\
\hline \multicolumn{3}{|l|}{ Actividad laboral } \\
\hline Estudiantes & 7 & 7 \\
\hline Dueña de casa & 55 & 55 \\
\hline Trabajadora & 38 & 38 \\
\hline \multicolumn{3}{|l|}{ Nivel Socioeconómico } \\
\hline Bajo (< U\$ 143 per cápita) & 68 & 68 \\
\hline Medio (entre U\$ 143 y 407 per cápita) & 32 & 32 \\
\hline
\end{tabular}

Tabla 3. Cálculo del riesgo que representa la percepción menor del estado nutricional por parte de la madre.

\begin{tabular}{|c|c|c|c|}
\hline & Mujeres & Hombres & Total \\
\hline Percepción menor & OR 23,22 & OR 1,69 & OR 2,53 \\
\hline que la medida & $P=0,001$ & $P=0,37$ & $P=0,0002$ \\
\hline Obesidad & (IC 95\%: 3,97-228,78) & (IC 95\%: 0,45-6,42) & (IC 95\%: 1,43-4,48) \\
\hline Percepción menor & OR 0,04 & OR 0,59 & OR 0,19 \\
\hline que la medida & $P=0,001$ & $P=0,37$ & $P=0,0002$ \\
\hline Sobrepeso & (IC 95\%: 0,004-0,251) & (IC 95\%: 0,15-2,20) & (IC 95\%: 0,07-0,51) \\
\hline
\end{tabular}


$60 \%$ de las madres de niños con MNPE los percibieron como más delgados (Figura 1).

Para la variable obesidad independiente del género, la percepción materna de que su hijo tuviese un menor grado de obesidad fue significativa (Odds Ratio (OR) 2,53; $\mathrm{p}=0,0002$ (IC 95\%: 1,43-4,48). En cambio, que las madres tuvieran una subestimación del grado de obesidad en niñas respecto al valor nutricional medido incrementó significativamente el riesgo en la variable obesidad $(\mathrm{OR}=$ 23,22; $\mathrm{p}=0,001$ (IC 95\%: 3,97-228,78) (Figura 2).

\section{Discusión:}

Los resultados presentados indican que existe una relación importante entre la alteración de la percepción materna del estado nutricional de sus hijos y el aumento constante de la obesidad en escolares.

En nuestro país la MNPE, tanto en población infantil como adolescente o adulta es un grave problema de Salud Pública. Chile es el país con mayor aumento de obesidad infantil, alcanzando prevalencias importantes a nivel mundial ${ }^{10}$. Un informe reciente nos sitúa como el segundo país con mayor prevalencia de obesidad en adultos entre los integrantes de la $\mathrm{OCDE}^{11}$. Por su parte la última ENS encontró un $74 \%$ de MNPE en niños de 8 a 12 años, especialmente, en familias con bajo nivel socioeconómico $^{12-13}$. En la población de nuestro estudio, el $68 \%$ corresponde a un estrato socio económico bajo pertenecientes a una de las comunas más vulnerables de nuestro país, como Alto Hospicio.

Este estudio revela que, por parte de la madre, existe una distorsión de la imagen de su hijo con mal nutrición por exceso, ya que en $60 \%$ de los casos tendió a percibirlo en un estado nutricional menor que el clasificado. Un estudio chileno ha establecido una distorsión similar de un $54,8 \% 9$ y un estudio mexicano la estableció en 98,8\% 8 . En relación al género se encontró que comparados con los niños, el riesgo de subestimar el grado de MNPE fue mucho mayor en niñas.

Se pudo observar que, en la población estudiada, existe una tendencia a presentar un aumento de sus factores de riesgo cardiovasculares como glicemia, colesterol total, presión arterial alterados y obesidad abdominal según perímetro de cintura.

La alteración de la percepción materna en relación al estado nutricional de su hijo puede influir en la escasa adhesión al tratamiento para disminuir el sobrepeso y obesidad de los escolares.

A la luz de estos resultados es importante establecer nuevas estrategias, de las actualmente implementadas. Aparte de las orientadas a los cambios y estilos de vida saludable, es necesario incorporar en el trabajo multidisciplinario de los equipos de Atención Primaria herramientas para el trabajo con las madres de escolares con mal nutrición por exceso, que permitan mejorar la percepción corporal de sus hijos.

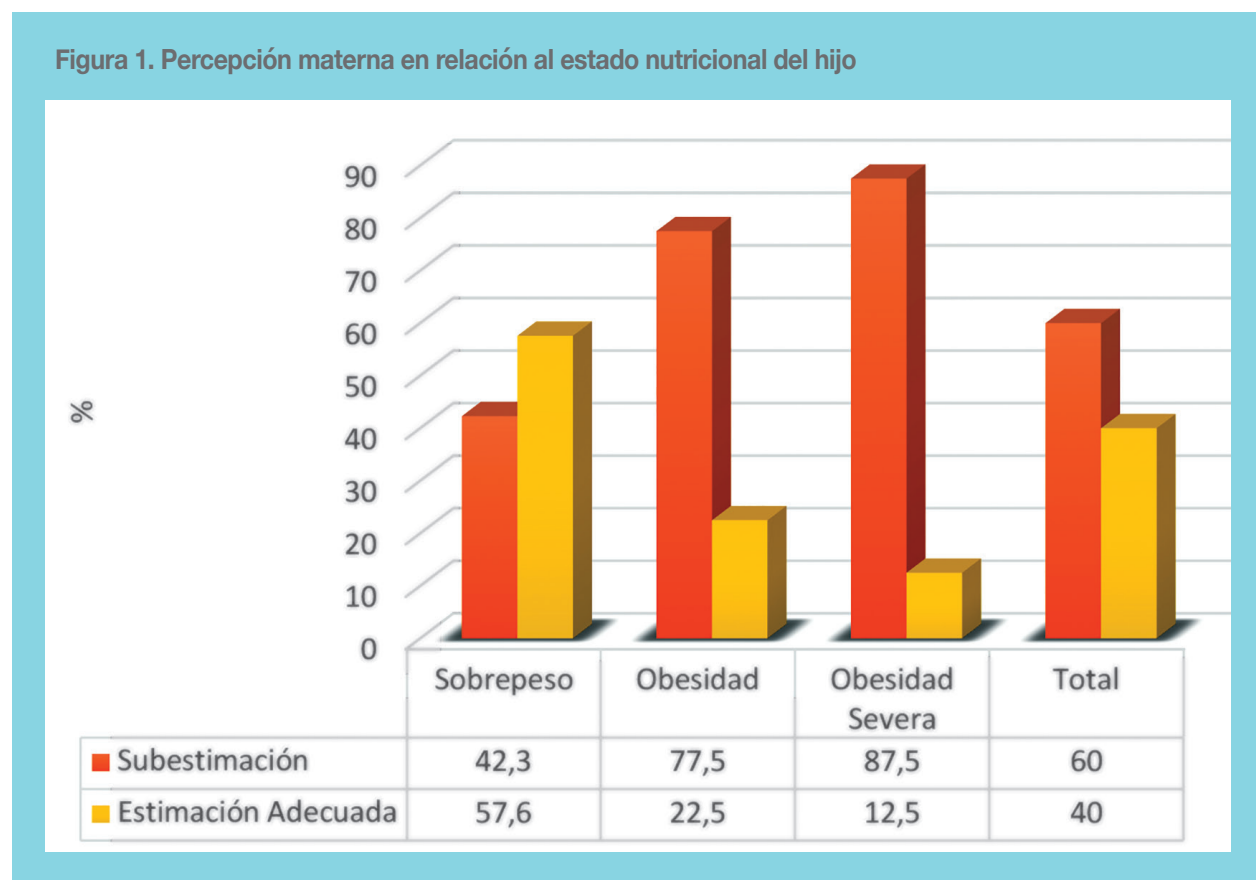




\section{PICTOGRAMA PERCEPCION MATERNA}

Pictograma Percepción Corporal Ninas
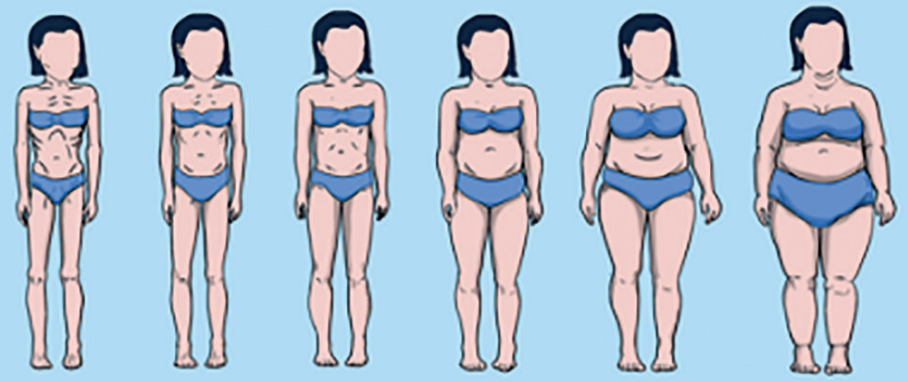

s.2

S.10eys.20e

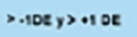

$2+106 y<+200$

$2 \cdot 20 t y<+30 e$

Pictograma Percepción Corporal Niños
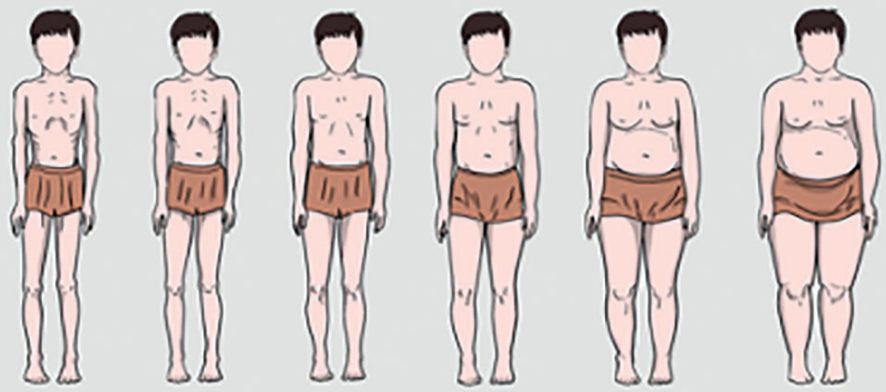

s.2

S. $100 y>-20 t>-10 t y>+106$

$z+10 t y<+200$

$2 \cdot 200 y<-306$

$2 \cdot 30 t$

puevits propus

\section{Conclusión:}

En relación a los factores de riesgo cardiovascular de los escolares estudiados con malnutrición por exceso, un alto porcentaje presenta obesidad abdominal, presión arterial alterada, colesterol total y glicemias en ayunas alteradas. Las madres en su mayoría tienen una alteración de la percepción sobre el estado nutricional de sus hijos, especialmente, en las niñas, la cual puede repercutir en forma significativa para el desarrollo y mantenimiento de la MNPE y, por consiguiente, ser un factor de riesgo cardiovascular clave a intervenir. 


\section{Referencias}

1.- BUENO M, SARRIA A. Obesidad. En: Pombo M, ed. Tratado de Endocrinología Pediátrica, 2da edición, Madrid: Díaz de Santos 1997:pp.1135- 1147.

2.- ORGANIZACIÓN MUNDIAL DE LA SALUD. (2007). obesidad. 2017, de OMS. [consultado abril 2018] Disponible en: http://www.who.int/dietphysicalactivity/childhood/es/.

3.- DEPARTAMENTO DE EPIDEMIOLOGIA. (2017). Encuesta nacional de salud. 2017. Minsal. [consultado Abril 2018] Disponible en: http://epi.minsal.cl/encuesta-ens/.

4.- DIETZ WH. Critical periods in childhood for the development of obesity. A m J ClinNutr 1994;59:955-959.

5.- OLIVARES S, BUSTOS N, MORENO X, LERA L, CORTEZ $\mathrm{S}$. Food and physical activity attitudes and practices in oobese children and their mothers in Santiago, Chile.RevChil Nutr.2006,33(2);170-9.

6.- UAUY R, ALBALA C, KAIN J. Obesity trends in Latin America; transiting from under to overweight. J Nutr.2001, 131(3);893-9.

7.- DECKELBAUM R, WILLIANS C, CHILDFOOD OBESITY, THE HEALTH ISSUE. Obes Res ClinPract.2001;9(4); 239-43.
8.- KARLA L, CHÁVEZ C, RODRIGUEZ J, GUZMÁN J, SEGOVIA J, ALTAMIRANO D, ET AL. Alteración de la percepción del estado nutricional por parte de preescolares: un factor de riesgo para obesidad y sobrepeso. Arch Argent Pediatr 2016;114(3);237-242.

9.- NUÑO M, HEVIA M, BUSTOS C, FLORENZANO R, FRITSCH R. Distorción de la imagen corporal en madres hacia sus hijos con sobrepeso u obesidad. Rev ChilNutr Vol. 44, $\mathrm{N}^{\mathrm{a}} 1,2017$.

10.-KOVALSKYS I, BAY L, RAUSCH C, BERNER E. Prevalence of obesity in a population of 10-19 year olds in a pediatric center. Rev ChilPed. 2005; 76(3): 324-325.

11.- OECD 2018. OECD Health Statistics 2018.Disponible en www.oecd.org/health/obesity-update.htm (consultado octubre 2018).

12.-ATALA E. Epidemiology of Obesity in Chile. Rev Med ClinCondes. 2012; 23(2): 117-123.

13.-ROZOWSKI J, ARTEAGA A. The problema of Obesity and Its Shocking Characteristics in Chile. Rev Med Chil. 1997; 10: 1217-1224. 